\title{
Suprathermal Electron Acceleration by an ICME-driven Quasi-perpendicular Shock on 2000 Feb 11
}

\author{
Fanjing Kong ${ }^{a, *}$ and Gang Qin ${ }^{b}$ \\ ${ }^{a}$ School of Physics and Electronics, North China University of Water Resources and Electric Power, \\ No. 136, Jinshuidong Road, Zhengzhou, China \\ ${ }^{b}$ School of Science, Harbin Institute of Technology, HIT Campus of University Town of Shenzhen, \\ Shenzhen, China \\ E-mail: kongfanjing@ncwu.edu.cn, qingang@hit.edu.cn
}

Using test-particle simulations we study the acceleration of suprathermal electrons at an ICMEdriven quasi-perpendicular shock on 2000 Feb 11 observed by Wind spacecraft. The downstream electron distribution in several energy channels from $\sim 0.3$ to $\sim 40 \mathrm{keV}$ are obtained assuming an initial distribution based on the observed upstream electron intensities. It is shown that in each energy channel the ratio of downstream to upstream intensities peaks at about $90^{\circ}$ pitch angle, and in each pitch angle direction the downstream electron energy spectral index is much larger than the theoretical index of diffusive shock acceleration. In addition, assuming the dominance of shock drift acceleration mechanism and the conservation of the phase space density before and after the acceleration, we find that the estimated drift length is proportional to electron energy but the drift time is almost energy independent. Furthermore, we construct a theoretical model based on SDA to describe the energy dependence of drift length and drift time. These results indicate the importance of SDA in the acceleration of electrons by quasi-perpendicular shocks, consistent with Yang et al. conclusion.

$37^{\text {th }}$ International Cosmic Ray Conference (ICRC 2021)

July 12th - 23rd, 2021

Online - Berlin, Germany

\footnotetext{
${ }^{*}$ Presenter
} 


\section{Introduction}

Shock acceleration is an important source for energetic particles in the heliosphere. The well-known diffusive shock acceleration (DSA) theory [1, 2, 4, 7], which successfully explains the power-law energy spectrum of cosmic rays, remains the dominant particle acceleration mechanism at collisionless shocks. Diffusive shock acceleration, contains drift and first-order Fermi acceleration. Shock drfit acceleration (SDA) is due to the gradient drift of particles along the direction of convection electric field. First-order Fermi aceleration (FFA) is due to the relative motion of scattering centers upstream and downstream regions of the shock. In addition, two other acceleration mechanisms also exists, i.e., stochastic acceleration (second-order Fermi) associated with the downstream turbulence, and shock surfing acceleration [e.g., 8] due to the cross-shock potential in the shock transition. Qin et al. [11] investigated the dominant acceleration mechanism by varing the shock geometry and turbulence level, and found that in the weak turbulence the particle acceleration process is FFA for parallel/quasi-parallel shocks and SDA for perpendicular/quasi-perpendicular shocks, and that SDA is more efficient compared to FFA.

In this article, we study numerically the acceleration of suprathermal electrons in the range of $\sim 0.3-40 \mathrm{keV}$ at the quasi-perpendicular shock on 2000 February 11 that was studied by Yang et al. [12]. Pitch angle distributions (PADs) for 12 energy channels, energy spectra for the parallel, perpendicular, and anti-parallel directions, and spectral indices for all pitch angles are obtained by solving the equation of motion of electrons using a backward-in-time test-particle method.

\section{Shock acceleration Model}

\subsection{Physical model}

A planar shock is located at $z=0$ with a thickness $L_{\text {th }}$. The plasma flows in the positive $z$-direction with the upstream and downstream speeds, $U_{1}, U_{2}$, respectively, in the shock frame. In this work, we use subscripts 1 and 2 to denote the upstream and downstream, respectively. In the shock transition the plasma speed is assumed to be in the form of

$$
U(z)=\frac{U_{1}}{2 s}\left\{(s+1)+(s-1) \tanh \left[\tan \left(-\frac{\pi z}{L_{\mathrm{th}}}\right)\right]\right\},
$$

where $s$ is the shock compression ratio. The equation of motion of test particles is given by

$$
\frac{d \boldsymbol{p}}{d t}=q[\boldsymbol{E}(\boldsymbol{r}, t)+\boldsymbol{v} \times \boldsymbol{B}(\boldsymbol{r}, t)],
$$

where $\boldsymbol{p}$ is the particle momentum, $\boldsymbol{v}$ is the particle velocity, $q$ is the electron charge, $t$ is time. The electric field $\boldsymbol{E}$ is the convection electric field $\boldsymbol{E}=-\boldsymbol{U} \times \boldsymbol{B}$. The total magnetic field consists of a background magnetic field and a turbulent magnetic field, and is given by

$$
\boldsymbol{B}\left(x^{\prime}, y^{\prime}, z^{\prime}\right)=\boldsymbol{B}_{0}+\boldsymbol{b}\left(x^{\prime}, y^{\prime}, z^{\prime}\right) .
$$

Note that the background magnetic field $\boldsymbol{B}_{0}$ is in the $x-z$ plane. The input parameters for the shock are shown in Table 1. The value of shock thickness $L_{\mathrm{th}}$ is set as $2 \times 10^{-6}$ au according to Yang et al. [12]. The turbulent magnetic field is given by

$$
\boldsymbol{b}\left(x^{\prime}, y^{\prime}, z^{\prime}\right)=\boldsymbol{b}_{\text {slab }}\left(z^{\prime}\right)+\boldsymbol{b}_{2 \mathrm{D}}\left(x^{\prime}, y^{\prime}\right)
$$




\begin{tabular}{ccc}
\hline \hline Parameter & Description & Value \\
\hline$\theta_{\mathrm{Bn}}$ & shock angle & $89^{\circ}$ \\
$V_{\mathrm{sh}}$ & shock speed & $682 \mathrm{~km} \mathrm{~s}^{-1}$ \\
$s$ & compression ratio & 2.87 \\
$B_{01}$ & upstream magnetic field & $7.0 \mathrm{nT}$ \\
$L_{\mathrm{th}}$ & shock thickness & $2 \times 10^{-6} \mathrm{AU}$ \\
$U_{1}$ & upstream speed & $248 \mathrm{~km} \mathrm{~s}^{-1}$ \\
$M_{\mathrm{A} 1}$ & upstream Alvén Mach number & 3.70 \\
\hline
\end{tabular}

Table 1: Input Parameters for the Shock

\begin{tabular}{ccc}
\hline \hline Parameter & Description & Value \\
\hline$\lambda$ & slab correlation length & $0.02 \mathrm{au}$ \\
$\lambda_{x}$ & 2D correlation length & $\lambda / 2.6$ \\
$E_{\text {slab }}: E_{2 \mathrm{D}}$ & two-component energy density ratio & $20: 80$ \\
$\left(b / B_{0}\right)^{2}{ }_{1}$ & upstream turbulence level & 0.25 \\
$\left(b / B_{0}\right)^{2}{ }_{2}$ & downstream turbulence level & 0.36 \\
$k_{\mathrm{b}}$ & break wavenumber & $10^{-6} \mathrm{~m}$ \\
$\beta_{\mathrm{i}}$ & inertial spectral index & $5 / 3$ \\
$\beta_{\mathrm{d}}$ & dissipation spectral index & 2.7 \\
\hline
\end{tabular}

Table 2: Input Parameters for the Turbulence

where $\boldsymbol{b}$ is a zero-mean turbulent magnetic field perpendicular to $\boldsymbol{B}_{0}$, and $\left(x^{\prime}, y^{\prime}, z^{\prime}\right)$ is the coordinate system with $z^{\prime}$ in the direction of $\boldsymbol{B}_{0}$. The turbulent magnetic field $\boldsymbol{b}$ is composed of slab and twodimensional (2D) components ["two-component" model, 3, 5, 9]. A periodic turbulence box with sizes $10 \lambda \times 10 \lambda$ and $25 \lambda$ for the $2 \mathrm{D}$ and slab components, respectively, is adopted in the simulations. The input parameters for the turbulence are shown in Table 2, where $k_{\mathrm{b}}$ represents the break number from the inerial to dissipation range.

\subsection{Numerical Model}

Based on the Wind/3DP observations, we simulate pitch angle distributions (PADs) of 12 energy channels with central energies $\sim 0.266,0.428,0.691,1.116,1.952,2.849,4.161,6.076$, $8.875,12.96,27.32$, and $39.50 \mathrm{keV}$. A backward-in-time test-particle method is used to simulate the PAD of a given energy channel $E_{i}(i=1,2,3, \ldots, 12)$ downstream of the shock. A total number of 30,000 electrons with an energy $E_{i}$ and a pitch angle $\mu_{j}$ are put into the downstream range $\left[z_{0}, z_{1}\right]$ at the initial time $t=0$, where $z_{0}=L_{\text {th }} / 2$ and $z_{1}=V_{\text {sh }} \Delta t \approx 2.7 \times 10^{-3}$ au with $\Delta t=10$ min. We take the spatial domain size in the $x, y, z$ directions to $x_{\text {box }}=y_{\text {box }}=10^{4} \lambda$ and $z_{\text {box }}=10^{3} \lambda$. The trajectory of each electron is followed using an adaptive step fourth-order Runge-Kutta method with a normalized accuracy of $10^{-9}$ until the simulation time $t_{\mathrm{acc}}=10 \mathrm{~min}$. After the numerical calculations, a few electrons whose energy is less than $0.1 E_{i}$ are discarded. The downstream pitch 
angle distribution, $f_{\mathrm{dn}}\left(E_{i}, \mu_{j}\right)$, for $E_{i}$ channel can be obtained as

$$
f_{\mathrm{dn}}\left(E_{i}, \mu_{j}\right)=\frac{1}{N_{i j}} \sum_{k=1}^{N_{i j}} f_{0}\left(E_{i k}, \mu_{j k}\right),
$$

where $N_{i j}$ is the number of test particles in the statistics, $f_{0}\left(E_{i k}, \mu_{j k}\right)$ is the initial distribution, and $E_{i k}$ and $\mu_{j k}$ are the $k$ th particle energy and pitch angle, respectively, when it is traced back to the initial time. The initial distribution is constructed by averaging the 3DP data in the time period of 23:20-23:30 UT before the shock arrival. Note that we employ linear interpolation in log-log space between the adjacent particle energies to calculate the value of $f_{0}\left(E_{i k}, \mu_{j k}\right)$. In addition, we do not consider wave excitation by the accelerated particles in this work.

\section{Drift length and time}

\subsection{Estimation from distribution functions with Liouville's theorem}

We apply Liouville's theorem to consider electron acceleration following Yang et al. [12]. It is assumed that the electron phase space density is conserved if the downstream accelerated electrons result from the upstream electrons due to SDA mechanism, i.e., $f_{2}\left(p_{2}\right)=f_{1}\left(p_{1}\right)$, where $p_{1}\left(p_{2}\right)$ and $f_{1}\left(f_{2}\right)$ are the electron momentum and phase space density in the upstream (downstream), respectively. The energy gain $\Delta E$ after the acceleration of upstream electrons with a momentum $p_{1}$ can be obtained.The electron drift length is then written as $L_{\mathrm{drift}}=\Delta E /(q|\boldsymbol{E}|)$, where $\boldsymbol{E}$ is the convection electric field. According to Jokipii [6], the gradient drift velocity at the shock front is $\boldsymbol{V}_{\mathrm{drift}}=\hat{\boldsymbol{e}}_{y} p v /(3 q)\left(B_{x 1} / B_{1}^{2}-B_{x 2} / B_{2}^{2}\right) \delta(z)$, where $B_{1}$ and $B_{2}$ are the background magnetic fields with their $x$-components $B_{x 1}$ and $B_{x 2}$ upstream and downstream of the shock, respectively. We integrate this equation for $z$ from $-L_{\text {th }} / 2$ to $L_{\text {th }} / 2$ to obtain the average gradient drift velocity

$$
\overline{\boldsymbol{V}}_{\mathrm{drift}}=\hat{\boldsymbol{e}}_{y} \frac{p v}{3 q L_{\mathrm{th}}}\left(\frac{B_{x 1}}{B_{1}^{2}}-\frac{B_{x 2}}{B_{2}^{2}}\right) .
$$

The drift time, $T_{\text {drift }}$, can be obtained by

$$
T_{\text {drift }}=\frac{L_{\text {drift }}}{\bar{V}_{\text {drift }}}
$$

\subsection{Calculation directly from simulations with a Monte-Carlo method}

Using numerical simulations for the trajectory of each test particle, we can calculate the total displacement in the drift direction and drift time when the gyro-orbits of particles are in the shock transition region. We obtain the average drift length and drift time by directly averaging over the upstream intensities of all test particles in the simulations.

\subsection{A theoretical model}

We construct a theoretical model of the electron drift length and drift time based on shock drift acceleration process. Since an electron's gyroradius is much smaller than the shock thickness $L_{\text {th }}$, it is assumed that an electron is accelerated by SDA when it is in the quasi-perpendicular 
shock transition range. When particles are in the shock transition range they would easily move downstream through solar wind convection, so the electron drift time $T_{\text {drift }}$ can be written as

$$
T_{\text {drift }}=\frac{L_{\mathrm{th}}}{2 U_{1}}+\frac{L_{\mathrm{th}}}{2 U_{2}},
$$

According to Equations (6), (7), and (8), the electron drift length $L_{\mathrm{drift}}$ can be calculated as

$$
L_{\mathrm{drift}}=T_{\mathrm{drift}} \overline{\mathrm{d}}_{\mathrm{drift}}=\frac{p v}{6 q}\left(\frac{1}{U_{1}}+\frac{1}{U_{2}}\right)\left(\frac{B_{x 1}}{B_{1}^{2}}-\frac{B_{x 2}}{B_{2}^{2}}\right) .
$$

It is found that the electron drift length $L_{\mathrm{drift}}$ is proportional to the electron kinetic energy $E$ if the relativistic effects are not considered, i.e.,

$$
L_{\text {drift }} \propto E .
$$

\section{Comparisons between simulations and observations}

Figure 1 shows the electron intensity versus pitch angle in the energy channels ranging from 0.266 to $39.50 \mathrm{keV}$ for the quasi-perpendicular shock event on $2000 \mathrm{Feb} 11$. The 10-minute average (23:20 UT-23:30 UT) upstream electron intensities for the observations (blue diamonds) show an anisotropic distribution with higher values in the parallel $\left(\mathrm{PA}=0^{\circ}\right)$ and anti-parallel $\left(\mathrm{PA}=180^{\circ}\right)$ magnetic field directions and lower values in the perpendicular $\left(\mathrm{PA}=90^{\circ}\right)$ direction except in the highest energy channel of $39.50 \mathrm{keV}$ (Figure 1(1)), where the intensity is not higher in the anti-parallel direction than in the perpendicular direction. The upstream average electron intensities also show that the anisotropy decreases with increasing electron energy. The downstream simulation results (black circles) are obtained using an initial distribution based on the 10-minute average upstream intensities from the observations. It is shown that in all these energy channels, the simulated downstream intensities increase much more compared with the upstream intensities around $90^{\circ}$ pitch angle. We also plotted the 10-minute average (23:34 UT-23:44 UT) downstream electron intenstities for the observations (red diamonds), which generally show an anti-parallel beam in the anti-parallel directions, except for the energy channels of $12.96 \mathrm{keV}$ and $27.32 \mathrm{keV}$ (Figure 1(j)$(\mathrm{k})$ ). We note that there is a poor agreement between the downstream simulations and observations, especially at energies below $8.875 \mathrm{keV}$, which can be attributed to the occurence of anti-parallel beams for the observations and weak shock acceleration efficiency for the simulations.

In Figure 2 we plot the ratio of the downstream to upstream average intensities for both the simulations (black circles) and observations (red diamonds). The simulated intensity ratio in all energy channels shows a peak at $\sim 80^{\circ}-100^{\circ}$ pitch angles. The observed intensity ratio also has a peak around $90^{\circ}$ pitch angle with the exception of $39.50 \mathrm{keV}$ energy channel. This indicates that at quasi-perpendicular shocks, the strongest acceleration occurs at pitch angles around $90^{\circ}$.

We compare the energy spectra of electrons in the directions parallel, perpendicular, and anti-parallel to the magnetic field for 10-minute average upstream/downstream electron intensities from the observations and downstream electron intensities from the simulations (not shown). It is found that the downstream intensities from both simulations and observations increase significantly compared to the initial upstream intensities in the perpendicular direction, and the index of powerlaw fit for these energy spectra are larger than the theoretical DSA index. In addition, we study the 


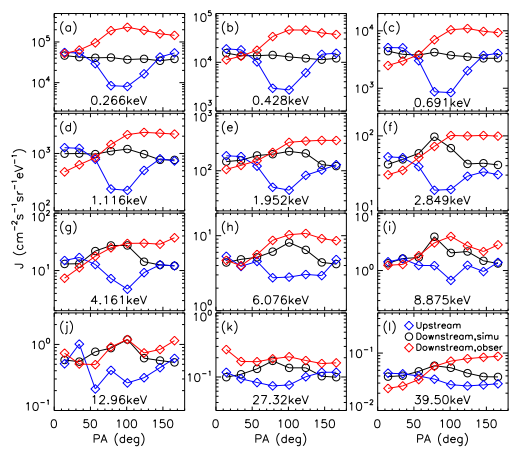

Figure 1: Electron differential intensity vs. pitch angle in the energy channels of $0.266-39.50 \mathrm{keV}$ denoted by (a)-(1). The blue and red diamonds correspond to 10-minute average upstream (23:20 UT-23:30 UT) and downstream (23:34 UT-23:44 UT) intensities from observations, respectively, and the black circles are downstream results from simulations using an initial distribution based on the observed upstream intensities.

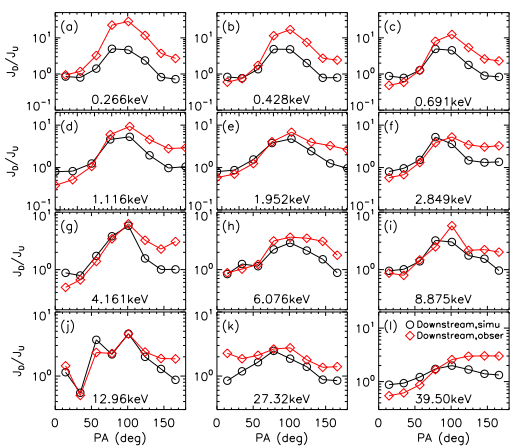

Figure 2: Ratio of the downstream to upstream average intensities for simulations (black circles) and observations (red diamonds).

variation of energy spectral index as a function of electron pitch angle (also not shown). It is found that in the quasi-perpendicular direction the downstream energy spectrum from both the observations and simulations are much softer compared to the upstream observations, indicating stronger shock acceleration in the perpendicular direction. The efficient acceleration in the perpendiuclar direction reveals the importance of SDA process at quasi-perpendicular shocks.

Figures 3(a) and (b) show the electron drift length $L_{\text {drift }}$ and drift time $T_{\text {drift }}$ as a function of energy, respectively. The results from simulations with Liouville's theorem and Monte-Carlo method are indicated by black and green circles (i.e., SIMU LT and SIMU MC), respectively. The results from observations with Liouville's theorem in Yang et al. [12] are indicated by red diamonds (i.e., OBSER LT). It is shown in Figure 3(a) that the drift length obtained from the simulations increases linearly with electron energy in log-log space with a slope of $\sim 1.1$ for both SIMU LT and SIMU MC, in agreement with that obtained from the observations using Liouville's theorem (OBSER LT) with a slope of 1.0. In Figure 3(b), a linear fit to the estimated drift time and electron energy in log-log space from the simulations yields a slope of 0.1 for both SIMU LT and SIMU MC, which agree approximately with that from the observations using Liouville's theorem (OBSER 


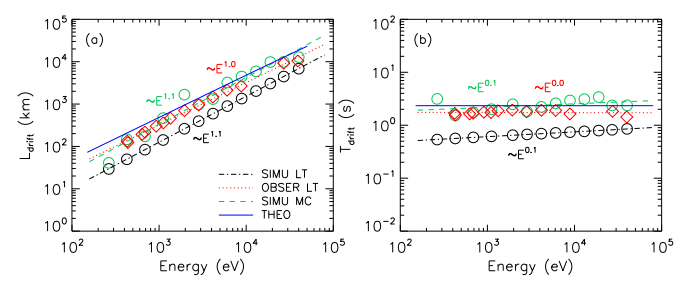

Figure 3: The drift length $L_{\text {drift }}$ and drift time $T_{\text {drift }}$ as a function of electron energy in (a) and (b), respectively. Red diamonds are results from observations with Liouville's theorem in Yang et al. [12]. Black and green circles are simulation results using Liouville's theorem and Monte-Carlo methods, respectively. Red dotted, black dash-dotted, and green dashed lines indicate linear fits to the data with red diamonds, black circles, and green circles, respectively. Blue solid lines indicate the theoretical results.

LT) with a slope of 0.0. It is therefore that the drift time almost does not vary with electron energy according to both the observations and simulations. The estimated drift length and drift time from simulations using Monte-Carlo method agree well with that from observations using Liouville's theorem, but they are larger than that from simulations using Liouville's theorem. It is probably due to the insufficient accuracy and less efficient acceleration of electrons in our numerical model. Furthermore, we use Equations (9) and (8) to calculate the theoretical results of electron drift length and drift time. It is shown that the electron drift length is proportional to electron energy and drift time is independent of electron energy (blue solid lines in Figure 3(a) and (b)). We find that the theoretical results agree well with the observations using Liouville's theorem.

\section{Summary}

We use test-particle numerical simulations of a backward-in-time method to study the acceleration of suprathermal electrons in the energy range of $\sim 0.3-40 \mathrm{keV}$ at a quasi-perpendicular shock event on 2000 February 11. The electron pitch angle distributions for 12 energy channels are obtained, and it is shown that the ratio of the downstream to upstream differential intensities peaks at about $\sim 90^{\circ}$ pitch angle, in good agreement with the spacecraft observations in Yang et al. [12]. In addition, it is found that the observed and simulated electron energy spectral index in each pitch angle direction downstream of the shock is significantly larger than the theoretical index of diffusive shock acceleration. This result indicates that shock drift acceleration plays an important role in the acceleration of electrons at quasi-perpendicular shocks as suggested by Yang et al. [12].

Considering the dominance of SDA process, Yang et al. [12] used Liouville's theorem to deal with the observational data, and showed that the electron drift length is approximately proportional to electron energy and drift time is almost independent of electron energy. We obtain the similar energy dependence of the drift length and drift time in the simulations by using Liouville's theorem. In addition, we obtain similar results directly calculated from the simulations using the Monte-Carlo method. Moreover, based on shock drift acceleration, we provide a theoretical model of electron drift length and drift time, which agree well with the observational results. This suggests that the proposed theoretical model can be used to explain the energy dependence of electron drift length and drift time found by the observations and simulations. Why our simulations show a 
weak acceelration and poor agreement with the observations is that we use a simple local shock acceleration model. The real condition, however, can be complicated, such as the complex shock geometry and magnetic field. On the other hand in the observations there are energetic particles from other sources, e.g., there possibly exist an anti-sunward-travelling beam of energetic electrons downstream of the shock in the observations which does not appear in our shock acceleration model.

\section{Acknowledgments}

This work was supported, in part, under grants NNSFC 41874206 and NNSFC 41574172. The work was carried out at National Supercomputer Center in Tianjin.

\section{References}

[1] Axford, W. I., Leer, E., \& Skadron, G, The acceleration of cosmic rays by shock waves, Proc. ICRC (Plovdiv) 2 (1977) 273

[2] Bell, A. R., The acceleration of cosmic rays in shock fronts-I., Mon. Not. R. Astron. Soc. 182 (1978) 147

[3] Bieber, J. W., Wanner, W., \& Matthaeus, W. H., Dominant two-dimensional solar wind turbulence with implications for cosmic ray transport, J. Geophys. Res. 101 (1996) 2511

[4] Blandford, R. D., \& Ostriker, J. P., Particle acceleration by astrophysical shocks, Astrophys. J. 221 (1978) L29

[5] Gray, P. C., Pontius, D. H., Jr., \& Matthaeus, W. H., Scaling of field-line random walk in model solar wind uctuations, Geophys. Res. Lett. 23 (1996) 965

[6] Jokipii, J. R., Particle drift, diffusion, and acceleration at shocks, Astrophys. J. 255 (1982) 716

[7] Krymsky, G. F., A regular mechanism for the acceleration of charged particles on the front of a shock wave, Dokl. Akad. Nauk SSSR 234 (1977) 1306

[8] Lee, M. A., Shapiro, V. D., \& Sagdeev, R. Z., Pickup ion energization by shock surfing, J. Geophys. Res. 101 (1996) 4777

[9] Matthaeus, W. H., Goldstein, M. L., \& Roberts, D. A., Evidence for the presence of quasi-twodimensional nearly incompressible fluctuations in the solar wind, J. Geophys. Res. 95 (1990) 20673

[10] Reames, D. V., Barbier, L. M., \& Ng, C. K., The Spatial Distribution of Particles Accelerated by Coronal Mass Ejection-driven Shocks, Astrophys. J. 466 (1996) 473

[11] Qin, G., Kong, F. J., \& Zhang, L. H., Effects of shock and turbulence properties on electron acceleration, Astrophys. J. 860 (2018) 3

[12] Yang, L., Wang, L., Li, G., et al., The Strongest Acceleration of $>40 \mathrm{keV}$ Electrons by ICMEdriven Shocks at 1 au, Astrophys. J. 853 (2018) 89 